\title{
Association of TyG index and TG/HDL-C ratio with arterial stiffness progression in a non-normotensive population
}

\author{
Zhiyuan Wu' ${ }^{1,3 \dagger}$, Di Zhou ${ }^{1 \dagger}$, Yue Liu' ${ }^{1}$, Zhiwei Li ${ }^{1}$, Jinqi Wang ${ }^{1}$, Ze Han ${ }^{1}$, Xinlei Miao ${ }^{1}$, Xiangtong Liu ${ }^{1}$, Xia Li ${ }^{2}$,
} Wei Wang ${ }^{3}$, Xiuhua Guo ${ }^{1,2^{*}}$ and Lixin Tao ${ }^{1,2^{*}}$ (D)

\begin{abstract}
Background: Cross-sectional studies have reported that insulin resistance (IR) is associated with arterial stiffness. However, the relationship between IR and arterial stiffness progression remains unclear. This study aims to evaluate the association of triglyceride glucose (TyG) index and triglyceride to high-density lipoprotein cholesterol (TG/HDL-C) ratio with arterial stiffness progression in a non-normotensive population.
\end{abstract}

Methods: A total of 1895 prehypertensive (systolic pressure $120-139 \mathrm{mmHg}$ or diastolic pressure $80-90 \mathrm{mmHg}$ ) or hypertensive (systolic pressure $\geq 140 \mathrm{mmHg}$ or diastolic pressure $\geq 90 \mathrm{mmHg}$ or using antihypertensive medication) participants were enrolled in 2013 and 2014, and followed until December 31, 2019. Arterial stiffness progression was measured by brachial-ankle pulse wave velocity (baPWV) change (absolute difference between baseline and last follow-up), baPWV change rate (change divided by following years), and baPWV slope (regression slope between examination year and baPWV).

Results: During a median follow-up of 4.71 years, we observed an increasing trend of baPWV in the population. There were linear and positive associations of the TyG index and TG/HDL-C ratio with the three baPWV parameters. The difference $(95 \% \mathrm{Cl})$ in baPWV change $(\mathrm{cm} / \mathrm{s})$ comparing participants in the highest quartile versus the lowest of TyG index and TG/HDL-C ratio were 129.5 (58.7-200.0) and 133.4 (52.0-214.9), respectively. Similarly, the evaluated baPWV change rates (cm/s/year) were 37.6 (15.3-60.0) and $43.5(17.8-69.2)$, while the slopes of baPWV were 30.6 (9.3-51.8) and 33.5 (9.0-58.0). The observed association was stronger in the hypertensive population.

Conclusion: Our study indicates that the TyG index and TG/HDL-C ratio are significantly associated with arterial stiffness progression in hypertensive population, not in prehypertensive population.

Keywords: Insulin resistance, Arterial stiffness, Hypertension, Triglyceride glucose index, TG/HDL-C ratio, Cohort study

*Correspondence: statguo@ccmu.edu.cn; taolixin@ccmu.edu.cn †Zhiyuan Wu and Di Zhou are first co-authors and drafted the manuscript together

${ }^{2}$ Department of Mathematics and Statistics, La Trobe University, Melbourne, Australia

Full list of author information is available at the end of the article

\section{Background}

Hypertension has caused a heavy economic burden worldwide, becoming a challenging public health issue. In 2010, 31.1\% of adults around the world were reported to have hypertension [1]. Among Chinese adults aged 35-75 years, nearly half are diagnosed with hypertension, the incidence is still steadily increasing, and the onset age is becoming younger [2]. Among hypertensive patients, arterial stiffness is a common vascular complication and

c) The Author(s) 2021. This article is licensed under a Creative Commons Attribution 4.0 International License, which permits use, sharing, adaptation, distribution and reproduction in any medium or format, as long as you give appropriate credit to the original author(s) and the source, provide a link to the Creative Commons licence, and indicate if changes were made. The images or other third party material in this article are included in the article's Creative Commons licence, unless indicated otherwise in a credit line to the material. If material is not included in the article's Creative Commons licence and your intended use is not permitted by statutory regulation or exceeds the permitted use, you will need to obtain permission directly from the copyright holder. To view a copy of this licence, visit http://creativeco mmons.org/licenses/by/4.0/. The Creative Commons Public Domain Dedication waiver (http://creativecommons.org/publicdomain/ zero/1.0/) applies to the data made available in this article, unless otherwise stated in a credit line to the data. 
is also an independent risk factor and predictor of other cardiovascular and cerebrovascular diseases [3, 4], such as coronary heart disease (CHD) and stroke. Therefore, it is of great importance to focus on the progression of arterial stiffness in the hypertensive population and identify the early related factors of arterial stiffness [5-7].

Disorders of glucose and lipid metabolism are a common pathophysiological feature accompanying patients with hypertension, while insulin resistance (IR) extensively participates in this biological process [8]. The hyperinsulinaemic-euglycaemic clamp is the gold standard for evaluating the status of IR [9]. However, this assessment process is expensive and complex and is not ideal for routine clinical monitoring. Recently, some novel and simple indicators have been reported to be reliable surrogate indexes of IR, such as the triglycerideglucose (TyG) index and the triglyceride to high-density lipoprotein cholesterol (TG/HDL-C) ratio. Compared with the hyperinsulinaemic-euglycaemic clamp, the TyG index has a high sensitivity of $96.5 \%$ and a specificity of $85.0 \%$ for the diagnosis of IR [10], which has also been reported to be associated with diabetes in Chinese population [11]. Many studies have found that these surrogate indexes are independent risk factors for some cerebrocardiovascular diseases [12-14]. Moreover, IR-related indexes are associated with arterial stiffness [15-19]. However, these studies are all based on general populations. Recently, Li et al. [20] found that the TyG index is positively associated with brachial-ankle pulse wave velocity (baPWV) in hypertensive patients. However, this study is a cross-sectional study without follow-up. Moreover, the association between the TG/HDL-C ratio and arterial stiffness remains unreported.

Therefore, we aimed to comprehensively investigate the association between the TyG index and TG/HDL-C ratio with arterial stiffness progression in prehypertensive and hypertensive populations based on a prospective cohort study.

\section{Methods}

\section{Study design and participants}

The Beijing health management cohort (BHMC) is an open cohort study established in 2008 in Beijing, China, with new individuals recruited annually. The BHMC study was conducted based on health examination populations from the Beijing Xiaotangshan Examination Center and Beijing Physical Examination Center. BHMC was designed to investigate the risk factors and biomarkers for metabolism-related diseases, and the recruited individuals were asked to take an annual health examination, including physical examination (anthropometry variables, blood pressures), face-to-face questionnaire survey (demographic variables, lifestyles, diseases history, medication history) and biochemical examination. Details of the study design have been described previously [21]. The arterial stiffness-related variables, such as baPWV and ankle brachial index (ABI), were available from 2013. Therefore, individuals taking health examinations in 2013 and 2014 were recruited in this current study as a baseline. Of 22,746 participants who underwent health examination at baseline, 2327 were confirmed to have prehypertension or hypertension. To minimize the possible influence of medication on glucose and lipid levels, 42 participants using antidiabetic agents and 52 participants using lipid-lowering agents were excluded. In addition, we excluded 101 participants with CHD, stroke or other cerebrocardiovascular diseases. Nineteen participants with any malignancy were excluded. Twenty-three participants from whom we were unable to collect the required data at baseline and 188 participants lost to follow-up, defined as without health examination records until 2019, were further excluded from the analyses. Finally, this study was restricted to a subset of 1895 participants with complete data, which were used in the final analyses, as shown in Fig. 1. Most of the participants (98\%) enrolled in this current study came from a work unit in Beijing, and there were a total of 407 units included.

This study was in accordance with the principles of the Declaration of Helsinki and approved by the Ethics Committee of Capital Medical University (grant number: 2020SY031). All participants provided written informed consent before taking part in this study.

\section{Data collection and definitions}

The demographic characteristics, lifestyle, and medication-related information were collected via a standard questionnaire by our trained staff, including age, sex, occupation, smoking status, drinking status, regular physical activity, diagnosis history of diseases and medication information. Occupation in this current study was classified into 'manager', 'technician or professional,' 'worker' and 'retired'. Smoking status was defined as 'current smoker,' 'former smoker' and 'never smoked'. Drinking status was defined as 'current drinking' and 'no current drinking. Physical activity was defined as having moderate or intense exercise ' $\geq 80 \mathrm{~min}$ per weak' and ' $<80$ min per week or none'. The physical and biochemical examination data were acquired from the electronic medical record system. Body mass index (BMI) was calculated as weight (in kilograms)/height ${ }^{\wedge} 2$ (in metres squared). Systolic blood pressure (SBP) and diastolic blood pressure (DBP) were presented as the average of two measurements on the right arm using a sphygmomanometer after resting for at least $10 \mathrm{~min}$. Based on the JNC-7 criteria [22], hypertension status 


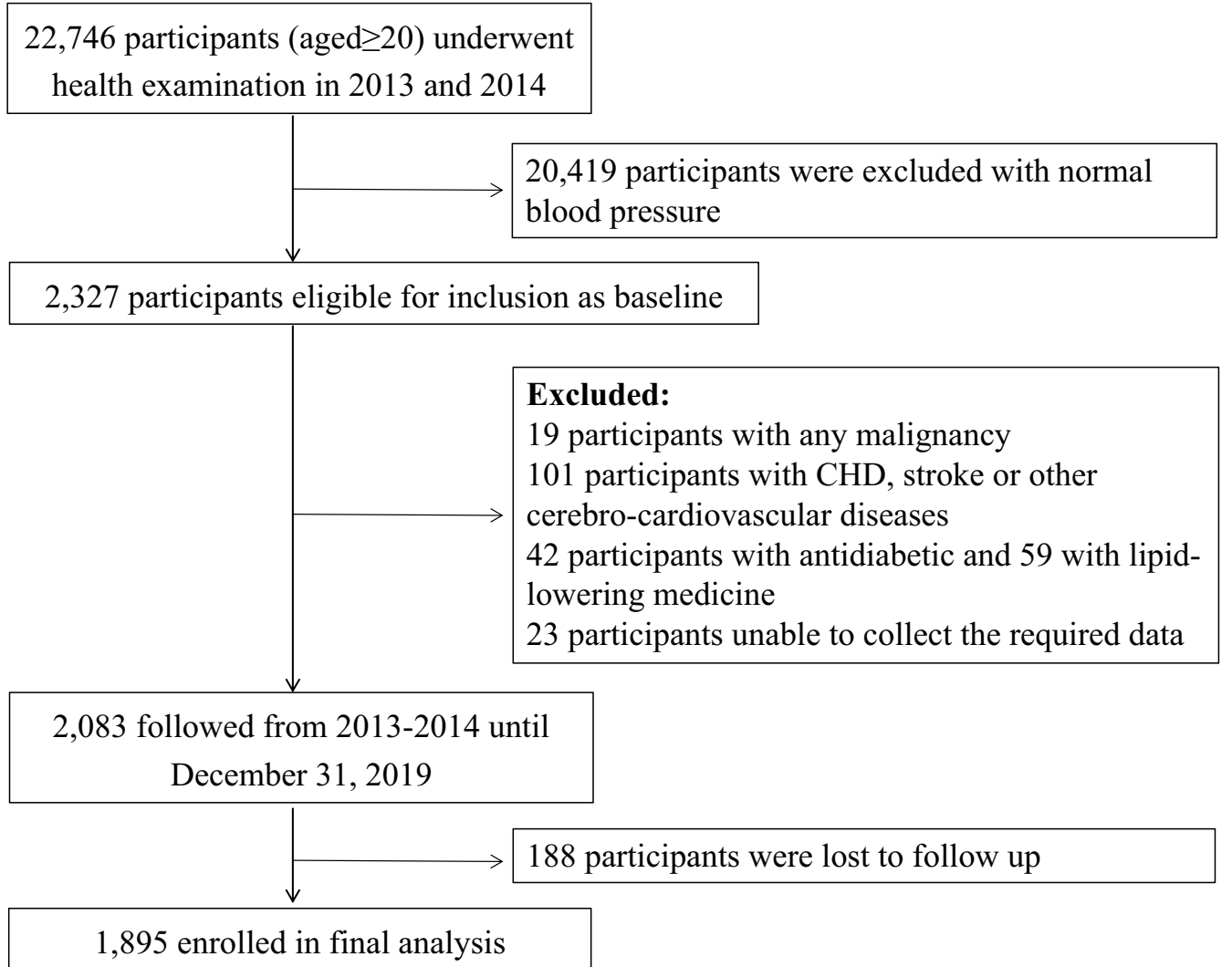

Fig. 1 Flow chart of the study population

was defined as $\mathrm{SBP} \geq 140 \mathrm{mmHg}$ or $\mathrm{DBP} \geq 90 \mathrm{mmHg}$ or use of any antihypertensive medication. Prehypertension status was defined as SBP of $120-139 \mathrm{mmHg}$ or DBP of $80-89 \mathrm{mmHg}$. The mean arterial pressure (MAP) was calculated as $1 / 3 * \mathrm{SBP}+2 / 3^{*} \mathrm{DBP}$. A higher vascular stiffness increases pulse pressure (PP), while PP could increase SBP and decrease DBP to a small extent. Therefore, the MAP level was adjusted in the following analyses.

Blood samples were stored and measured in the central laboratory of Beijing Xiaotangshan Examination Center or Beijing Physical Examination Center using the Olympus Automatic Biochemical Analyser (Hitachi 747; Tokyo, Japan). Serum total cholesterol (TC), triglyceride (TG), highdensity lipoprotein cholesterol (HDL-C), and low-density lipoprotein cholesterol (LDL-C) were measured with the enzymatic colour metric method. The estimated glomerular filtration rate (eGFR) was calculated using the Chronic Kidney Disease Epidemiology Collaboration (CKD-EPI 2009) serum creatinine equation [23]. Fasting blood glucose (FBG) was defined as the glucose concentrations before breakfast after overnight fasting (no food, except drinking water, for at least 8-10 h), while two-hour postprandial blood glucose (PBG) was measured $2 \mathrm{~h}$ after the beginning of fixed meals through finger blood tests. Diabetes was defined as FBG $\geq 7.0 \mathrm{mmol} / \mathrm{L}, \mathrm{PBG} \geq 11.1 \mathrm{mmol} / \mathrm{L}$, glycated haemoglobin $(\mathrm{HbA1c}) \geq 6.5 \%$ or the use of any glucose-lowering medication according to the American Diabetes Association [24]. According to the Guidelines on Prevention and Treatment of Dyslipidaemia for Chinese Adults [25], dyslipidaemia was defined as $\mathrm{TG} \geq 2.3 \mathrm{mmol} / \mathrm{L}, \mathrm{TC} \geq 6.2 \mathrm{mmol} / \mathrm{L}$, LDL-C $\geq 4.1 \mathrm{mmol} / \mathrm{L}, \mathrm{HDL}-\mathrm{C}<1.0 \mathrm{mmol} / \mathrm{L}$, or any lipidlowering medication. The TyG index was denoted as $\ln [\mathrm{TG}$ $(\mathrm{mm} / \mathrm{L})$ ) fasting glucose $(\mathrm{mm} / \mathrm{L}) / 2$ ] . The TG/HDL-C ratio was calculated as TG $(\mathrm{mm} / \mathrm{L})$ divided by HDL-C $(\mathrm{mm} / \mathrm{L})$.

\section{Assessment of baPWV}

baPWV is a simple, noninvasive, automatic and widely used method in clinical practice and large populationbased studies. The baPWV was measured with an Omron Colin BP-203RPE III device (Omron Health Care, Kyoto, Japan). After more than 5 min of rest in the supine position, 4 cuffs were wrapped around the bilateral brachia and ankles and then connected to a plethysmographic sensor and oscillometric pressure sensor. Semiconductor pressure sensors were used to assess the transmission time between the initial rises in both the brachial and tibial artery waves to record the pressure waveform. 
The distance between sampling points of baPWV was determined based on the height of the subjects. The time interval between the wave front of the brachial waveform and the ankle waveform was expressed as the time interval between the brachium and ankle $(\Delta \mathrm{Tba})$. The final baPWV was calculated as

$$
\mathrm{baPWV}=(\mathrm{La}-\mathrm{Lb}) / \Delta \mathrm{Tba}
$$

La was the path length from the suprasternal notch to the ankle, and Lb was the path length from the suprasternal notch to the brachium using the following equations:

$$
\mathrm{La}=0.8129 \times \text { height of the participant (in } \mathrm{cm})+12.328
$$

$\mathrm{Lb}=0.2195 \times$ height of the participant $($ in $\mathrm{cm})-2.0734$

The detailed process has been described in a previous study [26]. The maximum value of baPWV on the left and right sides was chosen as the final result of baPWV. The baPWV was measured at baseline and at the follow-up visit. The baPWV change was calculated as the baPWV value at baseline minus the baPWV value at the last visit during follow-up. The baPWV change rate was calculated as the value of baPWV change divided by the time distance between baseline and last visit. The slope of baPWV was defined as the linear regression slope with examination year as the independent variable and multiple baPWV measurements as the dependent variable.

\section{Statistical analysis}

Baseline characteristics are presented as the mean (standard deviation, SD), median [interquartile range, IQR] or number (percentage), as appropriate. Differences in baPWV change, baPWV change rate and slope of baPWV among groups were compared using the Kruskal-Wallis test.

Multivariate linear regression models were used to estimate the association of the TyG index and TG/HDL-C ratio with the absolute change, change rate and slope of baPWV. The TyG index and TG/HDL-C ratio were both analysed as continuous variables and categorized into quartiles. To adjust for potential confounding factors, three models were established as follows: model 1 adjusted for age and sex; model 2 adjusted for age, sex, BMI, smoking status, drinking status, physical activity, diabetes, dyslipidaemia, baPWV at baseline, and MAP at baseline and last follow-up; model 3 further adjusted for FBG (HDL-C if TG/HDL-C ratio analysed), triglyceride, PBG, LDL-C, eGFR, uric acid, homocysteine, and use of antidiabetic, lipid-lowering, or antihypertensive medications at baseline and follow-up. The regression coefficient and its 95\% confidence interval (CI) are presented. Spearman's correlation analyses of the TyG index and TG/
HDL-C ratio with other common cardiometabolic risk factors were performed. To identify the interaction of insulin resistance indexes and other variables, the interactive terms were tested in the model.

All of the analyses presented above were conducted using $\mathrm{R}$ software (version 3.6.3). The difference was considered statistically significant at two-sided $\mathrm{P}<0.05$.

\section{Results}

The final analysis included 1,895 individuals. During the follow-up period, a total of 986 recruited individuals attended health examinations twice, 612 had three examinations and 297 had four or five examinations. The mean age of the population was $61.90 \pm 12.75$ years, and 1,477 $(77.9 \%)$ were men. At baseline, 1,013 (53.5\%) participants were diagnosed with hypertension, and 882 (46.5\%) were diagnosed with prehypertension, among which 335 participants progressed to hypertension at the last visit of follow-up. The median values of the baPWV change, the baPWV change rate and the slope of baPWV were $46 \mathrm{~cm} / \mathrm{s}, 9 \mathrm{~cm} / \mathrm{s} /$ year and 8.11 in the whole population, respectively, as shown in Table 1 . The detailed characteristics according to the quartiles of the TyG index and TG/ HDL-C ratio are presented in Additional file 1: Table S1 and Table S2.

We observed linear and positive associations of the TyG index and TG/HDL-C ratio with the baPWV change, baPWV change rate and slope of baPWV, as shown in Fig. 2. In the fully adjusted model (model 3), a one-unit increase in the TyG index was associated with a $149.6 \mathrm{~cm} / \mathrm{s}$ increase in baPWV change, a $40.4 \mathrm{~cm} / \mathrm{s} /$ year increase in the baPWV change rate, and a $34.9 \mathrm{~cm} / \mathrm{s}$ increase in the baPWV slope. The average increases of baPWV change, change rate and slope, comparing participants in the highest quartile versus the lowest of the TyG index, were $129.5 \mathrm{~cm} / \mathrm{s}$ ( $\mathrm{P}$ for trend: $<0.001$ ), $37.6 \mathrm{~cm} / \mathrm{s} /$ year ( $\mathrm{P}$ for trend: $<0.001$ ), and 30.6 ( $\mathrm{P}$ for trend: 0.002 ), respectively. Similarly, a one-unit increase in the TG/ $\mathrm{HDL}-\mathrm{C}$ ratio was associated with a $37.1 \mathrm{~cm} / \mathrm{s}$ increase in baPWV change, a $9.8 \mathrm{~cm} / \mathrm{s} /$ year increase in the baPWV change rate, and an $8.0 \mathrm{~cm} / \mathrm{s}$ increase in the baPWV slope. The average increases in baPWV change, change rate and slope, comparing participants in the highest quartile versus the lowest quartile of the TG/HDL-C ratio, were $133.4 \mathrm{~cm} / \mathrm{s}$ ( $\mathrm{P}$ for trend: 0.002 ), $43.5 \mathrm{~cm} / \mathrm{s} /$ year ( $\mathrm{P}$ for trend: 0.001 ), and 33.5 ( $\mathrm{P}$ for trend: 0.004 ), respectively. The detailed regression results are shown in Tables 2 and 3. The distributions of baPWV change, baPWV change rate and slope of baPWV among the quartile groups according to the TyG index are shown in Fig. 3A-C. The distributions of baPWV parameters among the quartile groups according to the TG/HDL-C ratio are shown in Fig. 3D-F. To identify the significant 
Table 1 Characteristics of the study population

\begin{tabular}{|c|c|}
\hline & Characteristics $(\mathrm{N}=1895)$ \\
\hline \multicolumn{2}{|l|}{ At baseline } \\
\hline Age (years) & $61.90(12.75)$ \\
\hline Sex (men, \%) & $1477(77.9)$ \\
\hline \multicolumn{2}{|l|}{ Occupation (n, \%) } \\
\hline Manager & $392(20.7)$ \\
\hline Technician or professional & $214(11.3)$ \\
\hline Worker & $187(9.9)$ \\
\hline Retired & $1102(58.1)$ \\
\hline $\mathrm{BMI}$ & $26.52(3.22)$ \\
\hline Physical activity (n, \%) & $964(50.9)$ \\
\hline $\begin{array}{l}\text { Smoking status: none/former/current } \\
(\mathrm{n}, \%)\end{array}$ & $1128(59.6) / 285(15.0) / 482(25.4)$ \\
\hline Current drinking $(n, \%)$ & $844(44.5)$ \\
\hline $\mathrm{SBP}(\mathrm{mmHg})$ & $139.64(12.69)$ \\
\hline $\mathrm{DBP}(\mathrm{mmHg})$ & $78.67(10.06)$ \\
\hline MAP (mmHg) & $98.99(8.60)$ \\
\hline Hypertension (n, \%) & $1013(53.5)$ \\
\hline Antihypertensive medication ( $n, \%)$ & $267(26.4)$ \\
\hline Diabetes $(n, \%)$ & $323(17.0)$ \\
\hline Dyslipidaemia (n, \%) & $773(40.8)$ \\
\hline $\mathrm{FBG}(\mathrm{mmol} / \mathrm{L})$ & $5.79(1.48)$ \\
\hline PBG (mmol/L) & $7.72(2.61)$ \\
\hline $\mathrm{HbA1c}(\%)$ & $5.94(0.82)$ \\
\hline Triglyceride (mmol/L) & $1.86(1.62)$ \\
\hline Total cholesterol (mmol/L) & $4.81(0.98)$ \\
\hline LDL-C (mmol/L) & $3.11(0.88)$ \\
\hline $\mathrm{HDL}-\mathrm{C}(\mathrm{mmol} / \mathrm{L})$ & $1.25(0.32)$ \\
\hline eGFR (mL/min per $\left.1.73 \mathrm{~m}^{2}\right)$ & $90.88(26.05)$ \\
\hline Uric acid $(\mu \mathrm{mol} / \mathrm{L})$ & $365.83(84.67)$ \\
\hline Homocysteine ( $\mu \mathrm{mol} / \mathrm{L})$ & $12.74(7.66)$ \\
\hline TyG index & $1.42[1.03,1.83]$ \\
\hline TG/HDL-C ratio & $1.22[0.78,1.96]$ \\
\hline baPWV (cm/s) & $1538.00[1392.00,1769.50]$ \\
\hline \multicolumn{2}{|l|}{ At last visit of follow up } \\
\hline $\mathrm{baPWV}(\mathrm{cm} / \mathrm{s})$ & $1603.00[1412.00,1815.50]$ \\
\hline Change of baPWV $(\mathrm{cm} / \mathrm{s})$ & $46.00[-251.00,309.50]$ \\
\hline Change rate of baPWV (cm/s/year) & $9.00[-57.22,64.29]$ \\
\hline Slope of baPWV & $8.11[-54.72,66.96]$ \\
\hline Hypertension (n, \%) & $1348(71.1)$ \\
\hline Antihypertensive medication ( $n, \%)$ & $562(41.7)$ \\
\hline Antidiabetic medication (n, \%) & $151(8.0)$ \\
\hline Lipid-lowering medication $(\mathrm{n}, \%)$ & $273(14.4)$ \\
\hline
\end{tabular}

Data are the mean (SD), median [IQR] or number (\%)

BMI: body mass index; SBP: systolic blood pressure; DBP: diastolic blood pressure; MAP: mean arterial pressure; FBG: fasting blood glucose; PBG: postprandial blood glucose; HbA1c: glycated haemoglobin; HDL-C: high-density lipoprotein cholesterol; LDL-C: low-density lipoprotein cholesterol; eGFR: estimated glomerular filtration rate; TyG: triglyceride glucose; baPWV: brachialankle pulse wave velocity interaction of the TyG index and TG/HDL-C ratio with other covariates, such as age, sex, BMI, diabetes, dyslipidaemia, kidney function and life habits, we tested all of the interaction terms in the fully adjusted model (if not stratified). We found that only the associations of the TyG index and TG/HDL-C ratio with baPWV parameters were significantly different between prehypertensive and hypertensive populations. The strength of the associations of the TyG index and TG/HDL-C ratio with arterial stiffness progression were dominant in the hypertensive population, as shown in Additional file 1: Table S3 and Fig. 4. In addition, we fitted the joint TyG index and TG/ HDL-C ratio in the fully adjusted model to evaluate the joint association with artery stiffness progression. There was a stronger association between the TyG index and the absolute change, change rate and slope of baPWV than the TG/HDL-C ratio, as shown in Additional file 1: Table S4.

The correlation coefficients of the TyG index and TG/ HDL-C ratio with other common cardiometabolic risk factors are described in Additional file 1: Figure S1. There were weak correlations of the TyG index and TG/HDL-C ratio with $\mathrm{BMI}, \mathrm{SBP}, \mathrm{DBP}, \mathrm{FBG}, \mathrm{PBG}, \mathrm{HbA1c}, \mathrm{TC}$, LDL$\mathrm{C}$, serum uric acid, eGFR and homocysteine (Spearman's coefficients less than 0.5), apart from TG and HDL-C.

\section{Discussion}

In this prospective cohort study, we found that a higher TyG index and TG/HDL-C ratio were associated with a higher risk of arterial stiffness progression in the hypertensive population, during a median follow-up of 4.71 years. The observed associations were still significant after adjusting for important confounding factors, including age, BMI, blood pressure, medication use, lifestyle habits, serum uric acid, serum homocysteine, eGFR, diagnosis of diabetes and dyslipidaemia, which are traditional cardiometabolic risk factors and are related to arterial stiffness progression. TyG index and TG/HDL-C ratio deserve more attention in clinical practice for preventing artery stiffness and other cerebrocardiovascular complications in the hypertensive population.

Artery stiffness is a severe adverse event in the hypertensive population. On the other hand, arterial stiffness can lead to other macro- and microvascular complications and a series of organic damages, such as cerebrovascular diseases and renal damage [3, 27-29]. Moreover, research has found that some biological factors precede arterial stiffness progression in patients with hypertension, such as the duration of diabetes mellitus [30] and sodium sensitivity or resistance [31]. Therefore, identifying early and reliable related factors associated with artery stiffness in the hypertensive population is of great importance. The TyG index and TG/HDL-C ratio have 

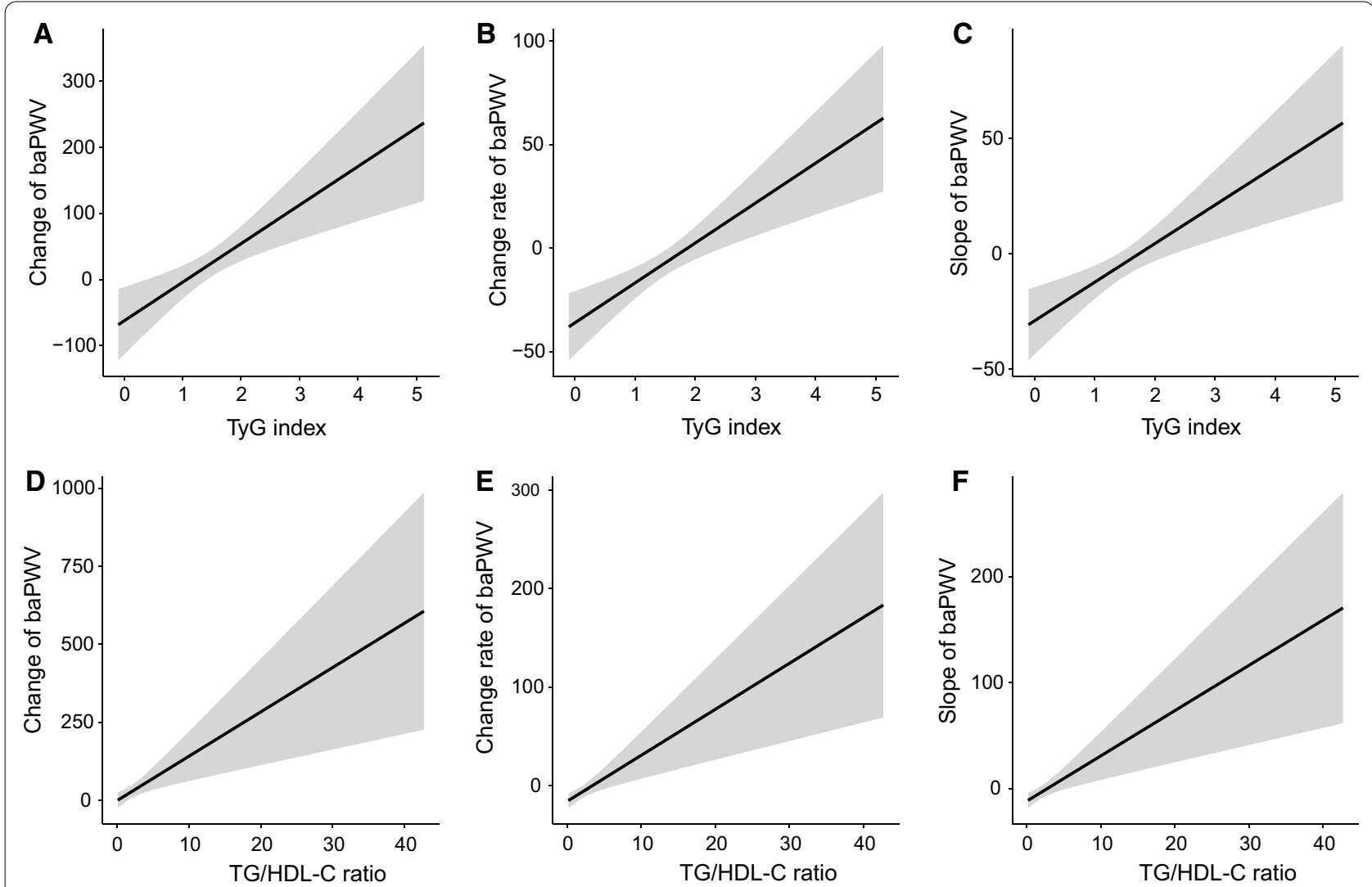

Fig. 2 The regression line of the TyG index and TG/HDL-C ratio with artery stiffness progression. A TyG index and baPWV change; B TyG index and baPWV change rate; $\mathbf{C T y G}$ index and slope of baPWV; D TG/HDL-C ratio and baPWV change; $\mathbf{E}$ TG/HDL-C ratio and baPWV change rate; $\mathbf{F}$ TG/HDL-C ratio and slope of baPWV

been reported to be associated with artery stiffness in the general population in previous studies. However, evidence in prehypertensive and hypertensive populations is scarce, except for the study by Li et al. [20], which reported that the TyG index is associated with evaluated baPWV in a hypertensive population based on a crosssectional design. Our study supplemented the evidence that the TyG index and TG/HDL-C ratio are associated with artery stiffness progression for the first time. We found that a higher TyG index and TG/HDL-C ratio were associated with an increased baPWV change, baPWV change rate and slope of baPWV in the hypertensive population based on a prospective cohort design.

The TyG index and TG/HDL-C ratio, have been of increasing interest at present. The TyG index and TG/ HDL-C ratio have high sensitivity and specificity for the diagnosis of insulin resistance compared with the hyperinsulinaemic-euglycaemic clamp and homeostatic model assessment in many populations [32, 33]. These two indicators have a high possibility of being easily applied in clinical practice for the early detection of IR, arterial stiffness and other diseases. Previous studies have also demonstrated the association of the TyG index with cardiovascular, cerebrovascular and other metabolic diseases. In 2014, Fedchuk et al. [34] measured five steatosis biomarkers, including the TyG index, fatty liver index (FLI), NAFLD liver fat score (NAFLD-LFS), hepatic steatosis index (HSI) and visceral adiposity index (VAI), and found that all five steatosis biomarkers could contribute to the early diagnosis of steatosis and were correlated with IR. In the Northern Shanghai Study, Zhao et al. [17] concluded that an elevated TyG index was significantly related to arterial stiffness and nephric microvascular damage, which supported the clinical application of the TyG index for the assessment of vascular damage. In recent years, several studies have also reported that the TyG index may predict several cardiovascular diseases, including acute coronary syndromes, symptomatic CHD and ischaemic stroke [35-39]. For the TG/ HDL-C ratio, in an 8-year Japan Diabetes Complications Study, Hirohito Sone et al. [40] evaluated conventional lipid variables, such as TG, non-HDLC, TC/HDL-C ratio, $\mathrm{LDL}-\mathrm{C} / \mathrm{HDL}-\mathrm{C}$ ratio, and TG/HDL-C ratio, for a relationship with $\mathrm{CHD}$. According to their analyses, all 
Table 2 Association of the TyG index with the absolute change, change rate and slope of baPWV

\begin{tabular}{|c|c|c|c|c|c|c|c|c|c|}
\hline & \multicolumn{3}{|c|}{ model 1} & \multicolumn{3}{|c|}{ model 2} & \multicolumn{3}{|l|}{ model 3} \\
\hline & $\beta$ & $95 \% \mathrm{Cl}$ & $P$ value & $\beta$ & $95 \% \mathrm{Cl}$ & $P$ value & $\beta$ & $95 \% \mathrm{Cl}$ & $P$ value \\
\hline \multicolumn{10}{|l|}{ Change of baPWV (cm/s) } \\
\hline TyG (continuous) & 32.034 & $4.642-59.425$ & 0.022 & 57.119 & $22.835-91.402$ & 0.001 & 149.582 & $90.823-208.342$ & $<0.001$ \\
\hline Quartile 2 (ref: quartile 1) & 59.745 & $10.509-108.982$ & 0.017 & 61.086 & $12.582-109.59$ & 0.014 & 76.297 & $28.63-123.964$ & 0.002 \\
\hline Quartile 3 & 85.613 & $36.416-134.81$ & 0.001 & 93.631 & 43.889-143.373 & $<0.001$ & 102.562 & $52.08-153.044$ & $<0.001$ \\
\hline Quartile 4 & 74.957 & $25.494-124.42$ & 0.003 & 98.555 & $37.584-159.526$ & 0.002 & 129.525 & $58.723-200.326$ & $<0.001$ \\
\hline P for trend & & & 0.002 & & & $<0.001$ & & & $<0.001$ \\
\hline \multicolumn{10}{|c|}{ Change rate of baPWV (cm/s/year) } \\
\hline TyG (continuous) & 11.892 & $3.415-20.368$ & 0.006 & 17.974 & 7.307-28.642 & 0.001 & 40.372 & $21.853-58.891$ & $<0.001$ \\
\hline Quartile 2 (ref: quartile 1) & 11.870 & $-3.385-27.125$ & 0.127 & 12.122 & $-2.986-27.229$ & 0.116 & 16.529 & $1.507-31.551$ & 0.031 \\
\hline Quartile 3 & 20.615 & $5.373-35.858$ & 0.008 & 22.642 & $7.149-38.135$ & 0.004 & 24.390 & $8.481-40.299$ & 0.003 \\
\hline Quartile 4 & 24.616 & $9.291-39.941$ & 0.002 & 30.750 & $11.76-49.741$ & 0.002 & 37.607 & $15.294-59.919$ & 0.001 \\
\hline P for trend & & & 0.001 & & & 0.001 & & & $<0.001$ \\
\hline \multicolumn{10}{|l|}{ Slope of baPWV } \\
\hline TyG (continuous) & 9.570 & $1.538-17.601$ & 0.020 & 14.238 & $4.121-24.355$ & 0.006 & 34.874 & $17.238-52.51$ & $<0.001$ \\
\hline Quartile 2 (ref: quartile 1) & 10.986 & $-3.469-25.441$ & 0.136 & 10.888 & $-3.439-25.215$ & 0.137 & 14.872 & $0.57-29.175$ & 0.042 \\
\hline Quartile 3 & 18.739 & $4.296-33.182$ & 0.011 & 20.092 & $5.399-34.785$ & 0.007 & 22.163 & 7.016-37.31 & 0.004 \\
\hline Quartile 4 & 20.126 & $5.604-34.647$ & 0.007 & 23.648 & $5.639-41.658$ & 0.010 & 30.576 & $9.332-51.82$ & 0.005 \\
\hline$P$ for trend & & & 0.004 & & & 0.003 & & & 0.002 \\
\hline
\end{tabular}

Model 1: Adjusted age and sex; Model 2: Age, sex, BMI, smoking status, drinking status, physical activity, diabetes, dyslipidaemia, baPWV at baseline, and MAP at baseline and follow-up; Model 3: Model 2 plus FBG, triglyceride, PBG, LDL-C, eGFR, uric acid, homocysteine, and use of antidiabetic, lipid-lowering, or antihypertensive medications at baseline and follow-up

Table 3 Association of TG/HDL-C ratio with the absolute change, change rate and slope of baPWV

\begin{tabular}{|c|c|c|c|c|c|c|c|c|c|}
\hline & \multicolumn{3}{|l|}{ Model 1} & \multicolumn{3}{|l|}{ Model 2} & \multicolumn{3}{|l|}{ Model 3} \\
\hline & $\beta$ & $95 \% \mathrm{Cl}$ & $P$ value & $\beta$ & $95 \% \mathrm{Cl}$ & $P$ value & $\beta$ & $95 \% \mathrm{Cl}$ & $P$ value \\
\hline \multicolumn{10}{|l|}{ Change of baPWV (cm/s) } \\
\hline TG/HDL-C ratio (continuous) & 6.795 & -1.193 to 14.784 & 0.096 & 4.934 & -3.684 to 13.552 & 0.062 & 37.057 & $7.85-66.264$ & 0.013 \\
\hline Quartile 2 (ref: quartile 1) & 70.320 & $21.037-119.604$ & 0.005 & 68.883 & $19.964-117.802$ & 0.006 & 75.317 & $24.101-126.534$ & 0.004 \\
\hline Quartile 3 & 83.153 & 34.299-132.007 & 0.001 & 89.453 & $38.544-140.362$ & 0.001 & 84.036 & $25.516-142.556$ & 0.005 \\
\hline Quartile 4 & 131.912 & $82.542-181.282$ & $<0.001$ & 142.938 & $78.525-207.35$ & $<0.001$ & 133.420 & $51.949-214.891$ & 0.001 \\
\hline P for trend & & & $<0.001$ & & & $<0.001$ & & & 0.002 \\
\hline \multicolumn{10}{|l|}{ Change rate of baPWV (cm/s/year) } \\
\hline TG/HDL-C ratio (continuous) & 2.587 & $0.115-5.06$ & 0.040 & 1.880 & -0.801 to 4.562 & 0.169 & 9.768 & $0.547-18.989$ & 0.038 \\
\hline Quartile 2 (ref: quartile 1) & 14.121 & -1.149 to 29.392 & 0.070 & 13.621 & -1.616 to 28.857 & 0.080 & 19.486 & $3.325-35.646$ & 0.018 \\
\hline Quartile 3 & 23.979 & $8.842-39.117$ & 0.002 & 25.543 & $9.687-41.399$ & 0.002 & 29.615 & $11.15-48.08$ & 0.002 \\
\hline Quartile 4 & 38.237 & $22.94-53.534$ & $<0.001$ & 40.369 & $20.307-60.431$ & $<0.001$ & 43.511 & $17.804-69.217$ & 0.001 \\
\hline P for trend & & & $<0.001$ & & & $<0.001$ & & & 0.001 \\
\hline \multicolumn{10}{|l|}{ Slope of baPWV } \\
\hline TG/HDL-C ratio (continuous) & 2.253 & -0.089 to 4.595 & 0.060 & 4.507 & $1.425-6.048$ & 0.045 & 7.973 & $0.801-22.317$ & 0.035 \\
\hline Quartile 2 (ref: quartile 1) & 12.212 & -2.264 to 26.687 & 0.098 & 11.300 & -3.154 to 25.754 & 0.126 & 15.465 & $0.078-30.852$ & 0.049 \\
\hline Quartile 3 & 21.636 & $7.287-35.986$ & 0.003 & 21.977 & $6.935-37.019$ & 0.004 & 24.296 & $6.715-41.877$ & 0.007 \\
\hline Quartile 4 & 33.088 & $18.587-47.589$ & $<0.001$ & 32.178 & $13.146-51.21$ & 0.001 & 33.496 & $9.02-57.972$ & 0.007 \\
\hline P for trend & & & $<0.001$ & & & $<0.001$ & & & 0.004 \\
\hline
\end{tabular}

Model 1: adjusted age and sex; Model 2: age, sex, BMI, smoking status, drinking status, physical activity, diabetes, dyslipidaemia, baPWV at baseline, and MAP at baseline and follow-up; Model 3: Model 2 plus HDL-C, triglyceride, PBG, LDL-C, eGFR, uric acid, homocysteine, and use of antidiabetic, lipid-lowering, or antihypertensive medications at baseline and follow-up 

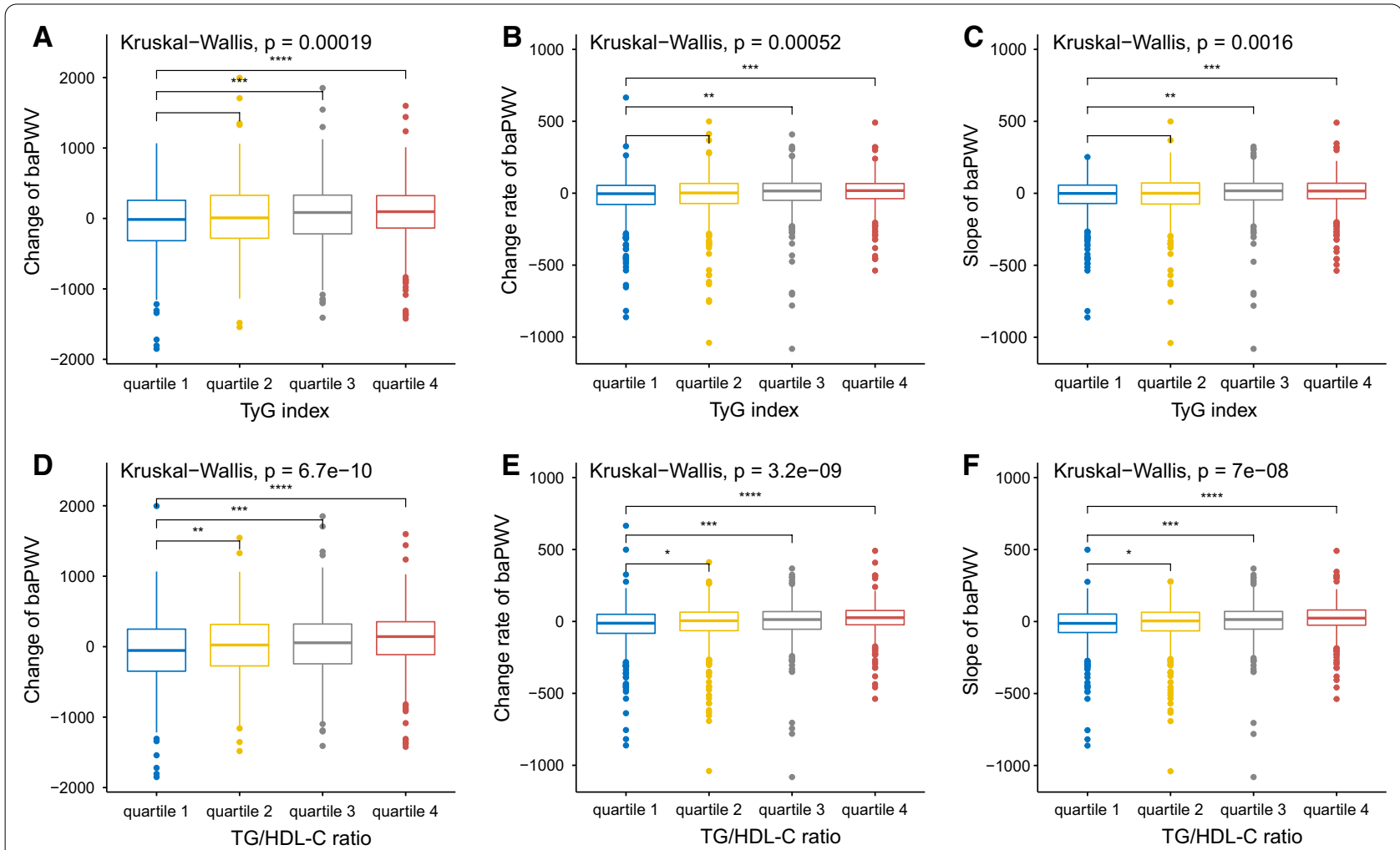

Fig. 3 The distributions of the absolute change, change rate and slope of baPWV among the quartile groups according to the TyG index (A-C) and TG/HDL-C ratio (D-F)

of these variables could predict CHD events in men and women. Marcello et al. [41] carried out a cross-sectional study to investigate the association between IR and TG/ HDL-C with CHD and concluded that HOMA-IR and TG/HDL-C are positively associated with CHD and may be useful as high-specificity indicators of CHD for risk stratification. These studies indicate that the TyG index and TG/HDL-C ratio are promising markers for future screening of metabolic diseases.

In the current study, we found that the evaluated TyG index and TG/HDL-C ratio were independently associated with the progression of artery stiffness in the hypertensive population, while a significant association was not observed in the prehypertensive population. In a previous study [42], blood pressure and the hypertensive state itself were reported to worsen the progression of arterial stiffness. In a review analysis [43], age and high blood pressure were the two main determinants of arterial stiffness. The findings in our study imply that the interaction between hypertensive status and insulin resistance leads to arterial stiffness progression, which means that people with hypertension should pay close attention to insulin resistance indexes to prevent artery stiffness. Although not completely elucidated, there are potential mechanisms linking the TyG index and TG/ HDL-C ratio with arterial stiffness. Insulin resistance is related with endothelial dysfunction, coagulation dysfunction, oxidative stress and inflammation, and cardiac concentric remodeling [44]. Hyperglycemia and hyperinsulinemia, usually accompanied by hyperlipidemia, are characterized by increased intracellular calcium concentration, increased collagen and advanced glycation end products, fibrosis and cellular hypertrophy, which reduce arterial elasticity through arterial remodeling especially in the hypertensive population, and lead to arterial stiffness $[45,46]$.

In addition, the TyG index and TG/HDL-C ratio, derived from fasting glucose, triglycerides and HDL$\mathrm{C}$, showed weak correlations with other components of metabolic syndrome, which implies that the TyG index and TG/HDL-C ratio might precede the incidence of the insulin resistance components [47]. Therefore, the TyG index and TG/HDL-C ratio may be early biomarkers of insulin resistance and other metabolic diseases, which warrants further validation in other studies. 

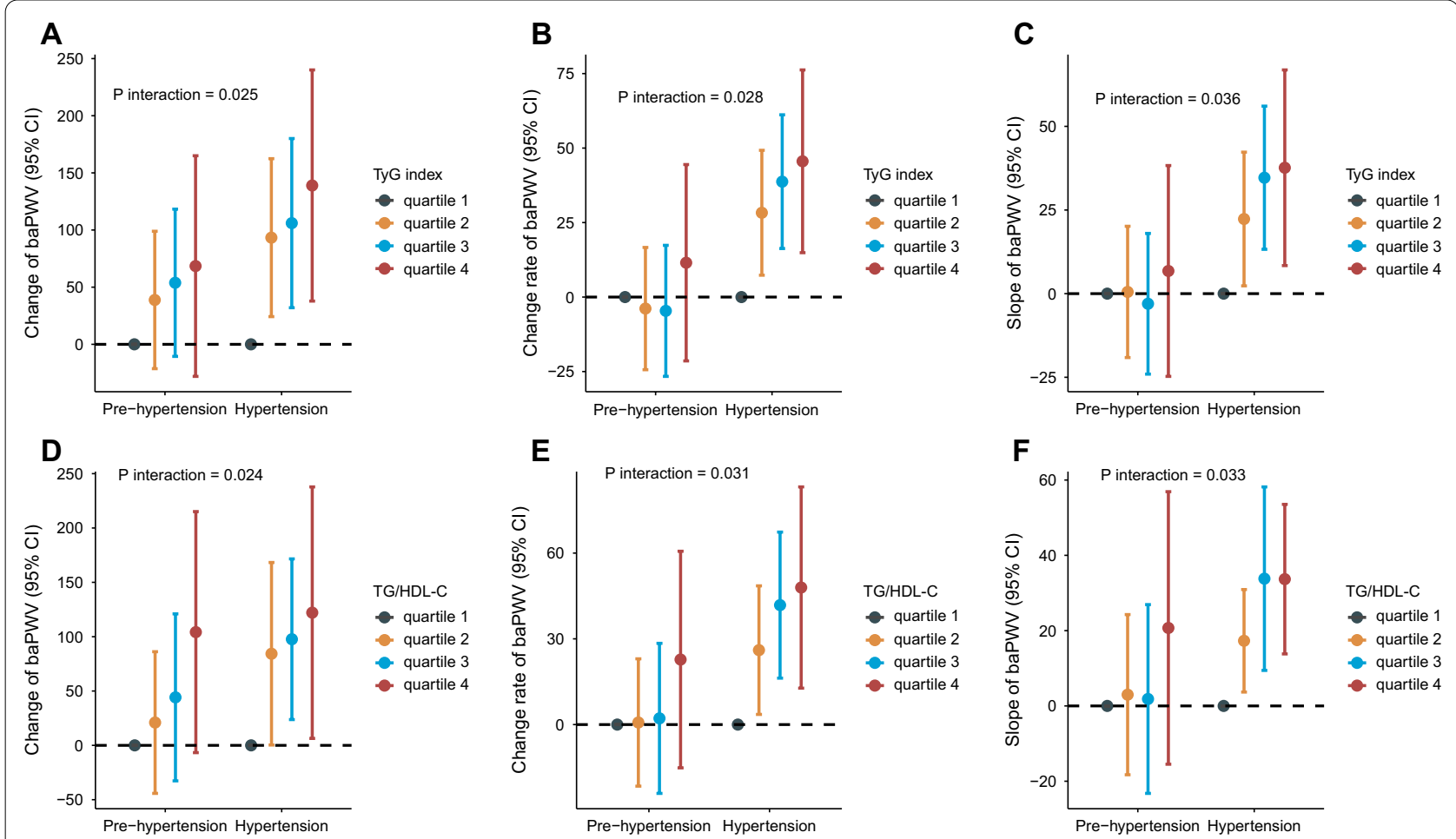

Fig. 4 Associations of the TyG index and TG/HDL-C ratio with the absolute change, change rate and slope of baPWV in prehypertensive and hypertensive populations*. *Adjusted for age, sex, BMI, smoking status, drinking status, physical activity, diabetes, dyslipidaemia, baPWV at baseline, FBG (HDL-C if TG/HDL-C ratio analysed), triglyceride, PBG, LDL-C, eGFR, uric acid, homocysteine, MAP at baseline and follow up, and use of antidiabetic, lipid-lowering, or antihypertensive medications at baseline and follow up

The strengths of the present study include the prospective cohort design to explore the association of the TyG index and TG/HDL-C ratio with the progression of artery stiffness in prehypertensive and hypertensive populations, the adjustment of the potential confounding factors, and handling the TyG index and TG/HDL-C ratio as both continuous variables and categorical variables to enhance the reliability of our findings. However, the results should be interpreted in the context of some limitations. First, the sample size was relatively small, and the 95\% CI of the estimated effect was wide. In the prehypertensive population, we only observed the evaluated tendency of baPWV change, baPWV change rate and slope of baPWV without statistical significance. The observed results in this single-centre study need further validation to generalize the associations in other populations and to evaluate the underlying biological mechanism between insulin resistance and artery stiffness progression. Second, the use of antihypertensive medication was considered in this analysis. However, we failed to collect the specific types of antihypertensive agents, given that different antihypertensive medications could have different influences on baPWV measurements. Data on antiplatelet medication were unavailable. Third, we analysed the association of the TyG index and TG/HDL-C ratio at baseline with artery stiffness progression in the current study, and evaluation of the dynamic TyG index and TG/ HDL-C ratio was needed with multiple measurements and related methods, such as trajectory analysis.

\section{Conclusion}

In summary, our findings indicate that the TyG index and TG/HDL-C ratio are significantly associated with arterial stiffness progression in hypertensive population, not in prehypertensive population. Monitoring the TyG index and TG/HDL-C ratio deserves more attention in clinical practice for the early prevention of arterial stiffness progression and other vascular complications of hypertension. 


\section{Abbreviations}

TyG: Triglyceride glucose index; TG/HDL-C: Triglyceride to high-density lipoprotein cholesterol ratio; baPWV: Brachial-ankle pulse wave velocity; IR: Insulin resistance; $\mathrm{BHMC}$ : Beijing health management cohort.

\section{Supplementary Information}

The online version contains supplementary material available at https://doi. org/10.1186/s12933-021-01330-6.

Additional file 1: Table S1. Baseline characteristics by quartile groups of TyG index. Table S2. Baseline characteristics by quartiles of TG/HDL-C ratio. Table S3. The association of insulin resistance indexes and arterial stiffness progression in prehypertensive and hypertensive populations. Table S4. Joint relationship of TyG index and TG/HDL-C ratio with the absolute change, change rate and slope of baPWV. Figure S1. The correlation of TyG index and TG/HDL-C ratio with the cardio-metabolic risk factors.

\section{Acknowledgements}

We thank all the staff and participants of the Beijing health management cohort for their invaluable contributions.

\section{Authors' contributions}

Study conception and design: XG, WW, and LT; Data collection: XM, YL, JW, and $\mathrm{ZH}$; Data analysis and interpretation: $\mathrm{ZW}, \mathrm{XL}, \mathrm{ZL}$, and $\mathrm{XL}$; Manuscript writing and reviewing: $Z W$, and DZ; Study supervision: $X G$, and LT. All authors read and approved the final manuscript.

\section{Funding}

Our work was funded by the National Natural Science Foundation of China (numbers: 81872708 and 82073668 to LX. Tao).

\section{Availability of data and materials}

The datasets used and/or analysed during the current study are available from the corresponding author (Dr. Lixin Tao) on reasonable request.

\section{Declarations}

\section{Ethics approval and consent to participate}

The study was approved by the Ethics Committees of Capital Medical University. All participants gave informed consent to participate before taking part. The approval number was 2020 SY031.

\section{Consent for publication}

Not applicable.

\section{Competing interests}

The authors declare that they have no competing interests.

\section{Author details}

${ }^{1}$ Beijing Municipal Key Laboratory of Clinical Epidemiology, Department of Epidemiology and Health Statistics, School of Public Health, Capital Medical University, Beijing, China. ${ }^{2}$ Department of Mathematics and Statistics, La Trobe University, Melbourne, Australia. ${ }^{3}$ School of Medical and Health Sciences, Edith Cowan University, Perth, Australia.

Received: 6 May 2021 Accepted: 28 June 2021

Published online: 06 July 2021

\section{References}

1. Mills KT, Bundy JD, Kelly TN, Reed JE, Kearney PM, Reynolds K, et al. Global disparities of hypertension prevalence and control: a systematic analysis of population-based studies from 90 countries. Circulation. 2016;134(6):441-50.
2. Lu J, Lu Y, Wang X, Li X, Linderman GC, Wu C, et al. Prevalence, awareness, treatment, and control of hypertension in China: data from 1.7 million adults in a population-based screening study (China PEACE Million Persons Project). Lancet. 2017;390(10112):2549-58.

3. Badji A, Sabra D, Bherer L, Cohen-Adad J, Girouard H, Gauthier CJ. Arterial stiffness and brain integrity: A review of MRI findings. Ageing Res Revi. 2019;53:100907.

4. Hughes TM, Wagenknecht LE, Craft S, Mintz A, Heiss G, Palta P, et al. Arterial stiffness and dementia pathology: Atherosclerosis Risk in Communities (ARIC)-PET Study. Neurology. 2018;90(14):e1248-56.

5. Ahmed TAN, Shams-Eddin H, Fathy MA, El-Naggar HM, Kishk YT. Subclinical left ventricular systolic dysfunction by two-dimensional speckletracking echocardiography and its relation to ambulatory arterial stiffness index in hypertensive patients. J Hypertens. 2020;38(5):864-73.

6. Aisu H, Saito M, Inaba S, Morofuji T, Takahashi K, Sumimoto T, et al. Association of worsening arterial stiffness with incident heart failure in asymptomatic patients with cardiovascular risk factors. Hypertens Res. 2017:40(2):173-80

7. Åström Malm I, Alehagen U, Blomstrand P, Dahlström U, De Basso R. Higher blood pressure in elderly hypertensive females, with increased arterial stiffness and blood pressure in females with the Fibrillin-1 2/3 genotype. BMC Cardiovasc Disord. 2020;20(1):180.

8. Sun X, Chen Y, Zeng Q, Huang X, Cai J. Reduction of leukocyte-derived $\mathrm{H}(2) \mathrm{S}$ linked to abnormal glycolipid metabolism in hypertensive subjects. Clin Exp Hypertens. 2017;39(5):427-34.

9. Muniyappa R, Lee S, Chen H, Quon MJ. Current approaches for assessing insulin sensitivity and resistance in vivo: advantages, limitations, and appropriate usage. Am J Physiol Endocrinol Metab. 2008;294(1):E15-26.

10. Guerrero-Romero F, Simental-Mendía LE, González-Ortiz M, et al. The product of triglycerides and glucose, a simple measure of insulin sensitivity. Comparison with the euglycemic-hyperinsulinemic clamp. J Clin Endocrinol Metab. 2010;95(7):3347-51.

11. Zhang $M$, et al. Cumulative increased risk of incident type 2 diabetes mellitus with increasing triglyceride glucose index in normal-weight people: The Rural Chinese Cohort Study. Cardiovasc Diabetol. 2017;16(1):30. https://doi.org/10.1186/s12933-017-0514-x

12. Fiorentino TV, Marini MA, Succurro E, Andreozzi F, Sesti G. Relationships of surrogate indexes of insulin resistance with insulin sensitivity assessed by euglycemic hyperinsulinemic clamp and subclinical vascular damage. BMJ Open Diabetes Research Care. 2019;7(1):e000911.

13. Wu Z, Wang J, Li Z, Han Z, Miao X, Liu X, Li X, Wang W, Guo X, Tao L. Triglyceride glucose index and carotid atherosclerosis incidence in the Chinese population: A prospective cohort study. Nutri Metab Cardiovasc Dis. 2021. https://doi.org/10.1016/j.numecd.2021.03.027.

14. Giannini C, Santoro N, Caprio S, Kim G, Lartaud D, Shaw M, et al. The triglyceride-to-HDL cholesterol ratio: association with insulin resistance in obese youths of different ethnic backgrounds. Diabetes Care. 2011;34(8):1869-74.

15. Lee SB, Ahn CW, Lee BK, Kang S, Nam JS, You JH, et al. Association between triglyceride glucose index and arterial stiffness in Korean adults. Cardiovasc Diabetol. 2018;17(1):41.

16. Won KB, Park EJ, Han D, Lee JH, Choi SY, Chun EJ, et al. Triglyceride glucose index is an independent predictor for the progression of coronary artery calcification in the absence of heavy coronary artery calcification at baseline. Cardiovasc Diabetol. 2020;19(1):34.

17. Zhao S, Yu S, Chi C, Fan X, Tang J, Ji H, et al. Association between macro- and microvascular damage and the triglyceride glucose index in community-dwelling elderly individuals: the Northern Shanghai Study. Cardiovasc Diabetol. 2019;18(1):95.

18. Won KB, Park GM, Lee SE, Cho IJ, Kim HC, Lee BK, et al. Relationship of insulin resistance estimated by triglyceride glucose index to arterial stiffness. Lipids Health Dis. 2018;17(1):268.

19. Irace C, Carallo C, Scavelli FB, De Franceschi MS, Esposito T, Tripolino C, et al. Markers of insulin resistance and carotid atherosclerosis. A comparison of the homeostasis model assessment and triglyceride glucose index. Int J Clin Pract. 2013;67(7):665-72.

20. Li M, Zhan A, Huang X, Hu L, Zhou W, Wang T, et al. Positive association between triglyceride glucose index and arterial stiffness in hypertensive patients: the China H-type Hypertension Registry Study. Cardiovasc Diabetol. 2020;19(1):139. 
21. Liu J, Zhao Z, Mu Y, Zou X, Zou D, Zhang J, et al. Gender differences in the association between serum uric acid and prediabetes: a six-year longitudinal cohort study. Int J Environ Res Public Health. 2018;15(7):1560.

22. Chobanian AV, Bakris GL, Black HR, Cushman WC, Green LA, Izzo JL Jr, Jones DW, Materson BJ, Oparil S, Wright JT, et al. The seventh report of the joint National Committee on prevention, detection, evaluation, and treatment of high blood pressure The JNC 7 report. JAMA. 2003;289(19):256072. https://doi.org/10.1001/jama.289.19.2560.

23. Levey AS, Stevens LA, Schmid CH, Zhang YL, Castro AF 3rd, Feldman HI, et al. A new equation to estimate glomerular filtration rate. Ann Intern Med. 2009;150:604-12.

24. American Diabetes Association. Standards of medical care in diabetes-2020 abridged for primary care providers. Clin Diabetes. 2020;38(1):10-38. https://doi.org/10.2337/cd20-as01.

25. Joint Committee for Developing Chinese Guidelines on Prevention and Treatment of Dyslipidemia in Adults. Chinese guidelines on prevention and treatment of dyslipidemia in adults. Chin J Epidemiol. 2007:35:390-419.

26. Yamashina A, Tomiyama H, Takeda K, Tsuda H, Arai T, Hirose K, Koji Y, Hori S, Yamamoto Y. Validity, reproducibility, and clinical significance of noninvasive brachial-ankle pulse wave velocity measurement. Hypertens Res. 2002;25(3):359-64.

27. Ruiz-Hurtado G, Ruilope LM. Microvascular injury and the kidney in hypertension. Hipertension y riesgo vascular. 2018;35(1):24-9.

28. Zuo J, Hu Y, Chang G, Chu SL, Tan I, Butlin M, et al. Relationship between arterial stiffness and chronic kidney disease in patients with primary hypertension. J Hum Hypertens. 2020;34(8):577-85.

29. Son WM, Sung KD, Bharath LP, Choi KJ, Park SY. Combined exercise training reduces blood pressure, arterial stiffness, and insulin resistance in obese prehypertensive adolescent girls. Clin Exp Hypertens. 2017:39(6):546-52.

30. Safar ME, Asmar R, Benetos A, Blacher J, Boutouyrie P, Lacolley P, et al. Interaction between hypertension and arterial stiffness. Hypertension. 2018;72(4):796-805.

31. He J, Huang JF, Li C, Chen J, Lu X, Chen JC, He H, Li JX, Cao J, Chen CS, Bazzano LA, Hu D, Kelly TN, Gu DF. Sodium sensitivity, Sodium resistance, and incidence of hypertension: a longitudinal follow-up study of dietary sodium intervention. Hypertension. 2021. https://doi.org/10.1161/HYPER TENSIONAHA.120.16758 (Epub ahead of print).

32. Mohd Nor NS, Lee S, Bacha F, Tfayli H, Arslanian S. Triglyceride glucose index as a surrogate measure of insulin sensitivity in obese adolescents with normoglycemia, prediabetes, and type 2 diabetes mellitus: comparison with the hyperinsulinemic-euglycemic clamp. Pediatr Diabetes. 2016;17:458-65. https://doi.org/10.1111/pedi.12303.

33. Vasques AC, Novaes FS, de Oliveira MS, et al. TyG index performs better than HOMA in a Brazilian population: a hyperglycemic clamp validated study. Diabetes Res Clin Pract. 2011;93:e98-100. https://doi.org/10.1016/j. diabres.2011.05.030

34. Fedchuk L, Nascimbeni F, Pais R, Charlotte F, Housset C, Ratziu V. Performance and limitations of steatosis biomarkers in patients with nonalcoholic fatty liver disease. Aliment Pharmacol Ther. 2014;40(10):1209-22

35. Alizargar J, Bai CH, Hsieh NC, Wu SV. Use of the triglyceride-glucose index (TyG) in cardiovascular disease patients. Cardiovasc Diabetol. 2020;19(1):8.

36. da Silva A, Caldas APS, Hermsdorff HHM, Bersch-Ferreira ÂC, Torreglosa $C R$, Weber $B$, et al. Triglyceride-glucose index is associated with symptomatic coronary artery disease in patients in secondary care. Cardiovasc Diabetol. 2019;18(1):89.

37. Sánchez-Îñigo L, Navarro-González D, Fernández-Montero A, PastranaDelgado J, Martínez JA. The TyG index may predict the development of cardiovascular events. Eur J Clin Invest. 2016;46(2):189-97.

38. Shi W, Xing L, Jing L, Tian Y, Yan H, Sun Q, et al. Value of triglycerideglucose index for the estimation of ischemic stroke risk: Insights from a general population. Nutr Metab Cardiovasc Dis. 2020;30(2):245-53.

39. Wang L, Cong HL, Zhang JX, Hu YC, Wei A, Zhang YY, et al. Triglycerideglucose index predicts adverse cardiovascular events in patients with diabetes and acute coronary syndrome. Cardiovasc Diabetol. 2020;19(1):80.

40. Sone H, Tanaka S, Tanaka S, limuro S, Ishibashi S, Oikawa S, et al. Comparison of various lipid variables as predictors of coronary heart disease in Japanese men and women with type 2 diabetes: subanalysis of the Japan Diabetes Complications Study. Diabetes Care. 2012;35(5):1150-7.

41. Bertoluci MC, Quadros AS, Sarmento-Leite R, Schaan BD. Insulin resistance and triglyceride/HDLc index are associated with coronary artery disease. Diabetol Metab Syndr. 2010;2:11.

42. Ato D, Sawayama T. Factors associated with high brachial-ankle pulse wave velocity in non-hypertensive and appropriately treated hypertensive patients with atherosclerotic risk factors. Vasc Health Risk Manag. 2017;13:383-92. https://doi.org/10.2147/NHRM.S144923 (Published 2017 Oct 10).

43. Laurent S, Boutouyrie P. Arterial stiffness and hypertension in the elderly. Front Cardiovasc Med. 2020;7:544302. https://doi.org/10.3389/fcvm.2020 544302 (Published 2020 Oct 29)

44. Markus MRP, Rospleszcz S, Ittermann T, et al. Glucose and insulin levels are associated with arterial stiffness and concentric remodeling of the heart. Cardiovasc Diabetol. 2019;18(1):145.

45. Prenner SB, Chirinos JA. Arterial stiffness in diabetes mellitus. Atherosclerosis. 2015;238(2):370-9.

46. Laurent S, Boutouyrie P. Arterial Stiffness and Hypertension in the Elderly. Front Cardiovasc Med. 2020;7:544302 (Published 2020 Oct 29).

47. Han T, Cheng Y, Tian S, et al. Changes in triglycerides and high-density lipoprotein cholesterol may precede peripheral insulin resistance, with 2-h insulin partially mediating this unidirectional relationship: a prospective cohort study. Cardiovasc Diabetol. 2016;15:154.

\section{Publisher's Note}

Springer Nature remains neutral with regard to jurisdictional claims in published maps and institutional affiliations.
Ready to submit your research? Choose BMC and benefit from:

- fast, convenient online submission

- thorough peer review by experienced researchers in your field

- rapid publication on acceptance

- support for research data, including large and complex data types

- gold Open Access which fosters wider collaboration and increased citations

- maximum visibility for your research: over 100M website views per year

At BMC, research is always in progress.

Learn more biomedcentral.com/submissions 